\title{
Transcription of the Immediate-Early Gene Arc in CA1 of the Hippocampus Reveals Activity Differences along the Proximodistal Axis That Are Attenuated by Advanced Age
}

\author{
Andrea L. Hartzell, ${ }^{1,2}$ Sara N. Burke, ${ }^{1,2}$ Lan T. Hoang, ${ }^{1,2}$ James P. Lister, ${ }^{1,2}$ Crystal N. Rodriguez, ${ }^{1,2}$ \\ and Carol A. Barnes ${ }^{1,2,3}$ \\ ${ }^{1}$ Evelyn F. McKnight Brain Institute, ${ }^{2}$ Arizona Research Laboratories Division of Neural Systems, Memory, Aging, and ${ }^{3}$ Departments of Psychology and \\ Neurology, University of Arizona, Tucson, Arizona 85724
}

The CA1 region of the hippocampus receives distinct patterns of afferent input to distal (near subiculum) and proximal (near CA2) zones. Specifically, distal CA1 receives a direct projection from cells in the lateral entorhinal cortex that are sensitive to objects, whereas proximal CA1 is innervated by cells in the medial entorhinal cortex that are responsive to space. This suggests that neurons in different areas along the proximodistal axis of CA1 of the hippocampus will be functionally distinct. The current experiment investigated this possibility by monitoring behavior-induced cell activity across the CA1 axis using Arc mRNA imaging methods that compared adult and old rats in two conditions: (1) exploration of the same environment containing the same objects twice (AA) or (2) exploration of two different environments that contained identical objects $(\mathrm{AB})$. The hypothesis was that $\mathrm{CA1}$ place cells should show field remapping in the condition in which environments were changed, but the extent of remapping was expected to differ between proximal and distal regions and between age groups. In fact, neurons in the proximal region of CA1 in adult animals exhibited a greater degree of remapping than did distal CA1 cells when the environment changed, suggesting that cells receiving input from the medial entorhinal cortex are more sensitive to spatial context. However, in old rats, there were no differences in remapping across the proximodistal CA1 axis. Together, these data suggest that distal and proximal CA1 may be functionally distinct and differentially vulnerable to normative aging processes.

\section{Introduction}

The hippocampus is critical for spatial memory (Morris et al., 1982), and it is well documented that its principal cells show firing rate increases at discrete locations of an environment (that is, the place field of the cell; O'Keefe and Dostrovsky, 1971). The composite activity of place fields forms a map (O'Keefe and Nadel, 1978), and, when an animal is returned to a familiar environment, the original map is retrieved (Thompson and Best, 1990). In contrast, when an animal is placed in a different environment, an independent map is generated ("remapping"; O'Keefe and Conway, 1978). In old animals, CA1 neurons have been reported to both fail to remap in a novel environment (Sava and Markus, 2008) and to remap inappropriately in a familiar environment (Barnes et al., 1997).

Within CA1, there are distinct patterns of input along the proximodistal axis (Amaral and Witter, 1995). Distal CA1 (near

\footnotetext{
Received Oct. 5, 2012; revised Dec. 14, 2012; accepted Dec. 21, 2012.

Author contributions: S.N.B. and C.A.B. designed research; A.L.H., S.N.B., L.T.H., and C.N.R. performed research; A.L.H., S.N.B., and J.P.L. analyzed data; A.L.H., S.N.B., and C.A.B. wrote the paper.

This work was supported by the McKnight Brain Research Foundation and National Institutes of Health Grants AG033460, AG036053, AG012609, and HHMI52003749. Additionally, we thank Andrew Maurer, Jeri Meltzer, Jacqueline Friel, Jonathan Ferng, Alan Mackey, Kathy Olson, Monica Chawla, Michael Montgomery, Michelle Carroll, and Luann Snyder for help with completing this manuscript.

The authors declare no competing financial interests.

Correspondence should be addressed to Carol A. Barnes, Life Sciences North Building, Room 355, P.0. Box 245115, University of Arizona, Tucson, AZ 85724-5115. E-mail: carol@nsma.arizona.edu.

DOI:10.1523/JNEUROSCI.4727-12.2013

Copyright $\odot 2013$ the authors $\quad 0270-6474 / 13 / 333424-10 \$ 15.00 / 0$
}

the subiculum) receives a direct projection from the objectresponsive lateral entorhinal cortex (LEC) (Deshmukh and Knierim, 2011) and perirhinal cortex (PRC) (Burke et al., 2012c; Deshmukh et al., 2012), whereas the space-responsive medial entorhinal cortex (MEC) (Fyhn et al., 2004) directly innervates proximal CA1 (near CA2). In line with these differences in afferent input, place field firing in proximal CA1 is more modulated by space relative to neurons in distal CA1 (Henriksen et al., 2010). Finally, the firing of CA1 neurons has been shown to be modulated by objects (Komorowski et al., 2009; Manns and Eichenbaum, 2009; Burke et al., 2011a), and this phenomenon appears to be particularly prevalent in distal areas of CA1 (Burke et al., 2011a). Whether the differential contributions of spatial versus nonspatial afferent input to CA1 are maintained during aging has not been examined directly and may provide insight into the integrity of cells that project to the hippocampus.

The current experiment investigated the extent to which activity in distal and proximal CA1 of adult and old rats was differentially affected by two episodes of object exploration in which the environment either remained the same or changed between epochs, using cellular compartment analysis of temporal activity by fluorescence in situ hybridization (catFISH) (Guzowski et al., 1999). catFISH uses the subcellular distribution of Arc mRNA to infer the activity history of large ensembles of neurons during two distinct $5 \mathrm{~min}$ epochs of behavior separated by $20 \mathrm{~min}$. Within minutes of principal neuron activation, Arc mRNA can be observed as intranuclear foci. Arc mRNA then translocates to the 
cytoplasm 15-20 min after cell firing (Guzowski et al., 1999). Therefore, neurons with $A r c$ in the nucleus indicate that the cell was active during the second epoch of behavior, whereas neurons with cytoplasmic Arc were active during the first epoch of behavior (Guzowski et al., 1999). Likewise, cells with both nuclear and cytoplasmic Arc were active during both epochs of behavior. It was hypothesized that the proportions of neurons active during both epochs of object exploration would vary along the proximodistal axis of CA1, and these differences would be affected by the distinct behavioral conditions and age groups.

\section{Materials and Methods}

Subjects and behavioral procedures. Twenty-four adult (7-9 months old) and 23 old (24-27 months old) male Fischer 344 rats (the National Institute on Aging colony at Charles River) were used. The rats were housed individually in Plexiglas guinea pig tubs $(23 \mathrm{~cm}$ wide $\times 20 \mathrm{~cm}$ tall $\times 44 \mathrm{~cm}$ deep) and maintained on a reversed $12 \mathrm{~h}$ light/dark cycle. All testing occurred during the dark phase of the light/dark cycle. Each rat was given ad libitum access to food and water for the duration of the experiment. Rats were handled for $10-15 \mathrm{~min} / \mathrm{d}$ for several days before testing.

All rats that explored objects in the current experiment participated in the spatial and visually cued versions of the Morris swim task (Morris et al., 1982) conducted in a tank $5.7 \mathrm{~m}$ in circumference and $0.5 \mathrm{~m}$ deep. This task has been described in detail previously (Barnes et al., 1996). To summarize, during the spatial version of the Morris swim task, all animals participated in six trials per day over 4 consecutive days. The rats completed three sets of two back-to-back trials per day, with $\leq 60 \mathrm{~s}$ rest between the two trials and $\sim 1 \mathrm{~h}$ of rest between sets. During these trials, a circular platform with a $16 \mathrm{~cm}$ diameter was hidden below the surface of water made opaque with nontoxic Sargent Art white powder paint and water temperature $\sim 22^{\circ} \mathrm{C}$. Rats were released from seven different start locations equally spaced around the perimeter of the tank. The start locations were pseudorandomized such that no rat was released from the same location on two consecutive trials. On the $2 \mathrm{~d}$ after the spatial trials, the rats participated in six cued visual trials per day in which the escape platform was raised above the surface of the water. The position of the platform changed between each trial to a different quadrant of the tank. Performance on the swim task was analyzed offline with a commercial software application (ANY-maze). Corrected integrated path length (CIPL) was calculated to ensure comparability of the rats' performances across different release locations and different swimming velocities. The CIPL is a measure of the cumulative distance from the escape platform over time corrected for the rat's swimming velocity and is equivalent to the cumulative search error (Gallagher et al., 1993).

After the Morris swim task, 23 rats (12 adult and 11 old) were randomly assigned to either the negative or positive control condition (see below). The remaining 12 adult and 12 old rats were randomly assigned and participated in one of two different variants of an object-recognition task ( $\mathrm{AA}$ or $\mathrm{AB}$ condition) in which rats were allowed to freely explore five distinct novel objects for two $5 \mathrm{~min}$ epochs separated by a $20 \mathrm{~min}$ rest in the home cage. In both the $\mathrm{AA}$ and $\mathrm{AB}$ conditions, the same five objects were explored during the two epochs of behavior; thus, the only variable that changed was the testing arena/room used for the second epoch of exploration (A or B). Additionally, both the $\mathrm{AA}$ and $\mathrm{AB}$ groups were habituated to the arenas for the $2 \mathrm{~d}$ directly preceding object exploration. For the AA condition, rats were habituated to an empty arena A $(60 \times 60$ $\mathrm{cm}$ ) for $10 \mathrm{~min} / \mathrm{d}$ in the same room. Rats that participated in the $A B$ condition were habituated to both arena $A$ and arena $B$ (also $60 \times 60 \mathrm{~cm}$ but differed from area $\mathrm{A}$ in both color and wall material and was located in a different room) for 10 min on 2 consecutive $\mathrm{d}$. For all $\mathrm{AB}$ rats, there was at least a 20 min delay between exposures to arena $A$ and arena $B$. Thus, arena $\mathrm{A}$ was used for both the $\mathrm{AA}$ and $\mathrm{AB}$ groups, but arena $\mathrm{B}$ was only used for the $\mathrm{AB}$ group.

Figure 1 shows a summary of the object-exploration procedure used for the $\mathrm{AA}$ and $\mathrm{AB}$ groups. On the day of the experiment, each rat was placed individually into arena A to freely explore five novel objects. After this initial exposure (epoch 1), the rat was returned to its home cage in
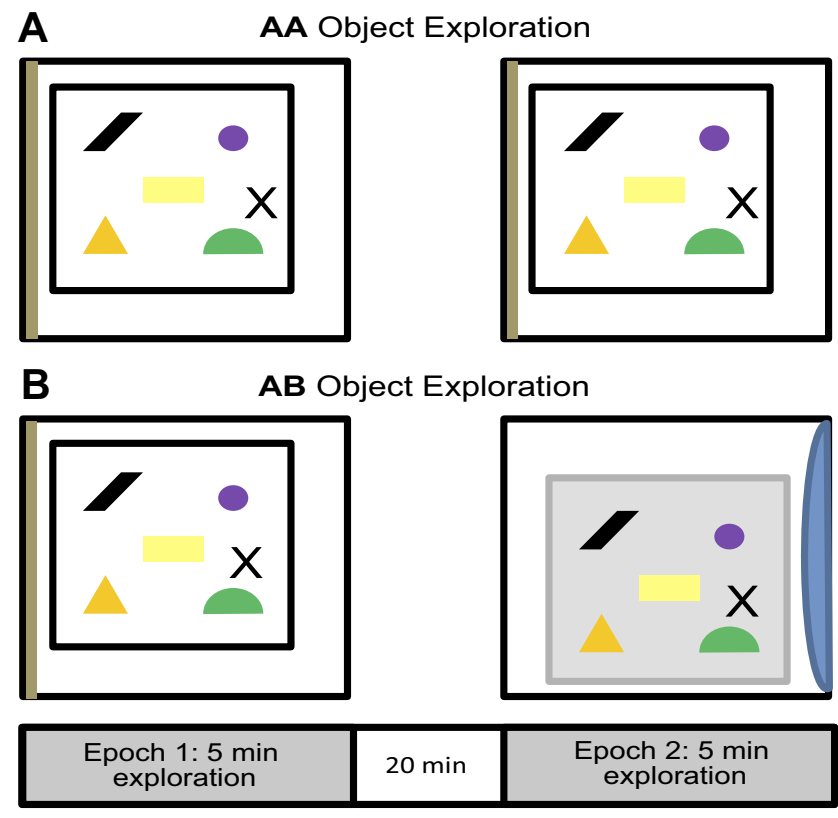

Figure 1. Schematic of exploration behavior. $\boldsymbol{A}, \boldsymbol{B}$, Diagram of the catFISH behavioral protocol showing the $A A$ (same environment and objects during both epochs of exploration) and $A B$ (different environment but same objects between epochs of exploration) conditions. Twelve adult and 12 old rats each spent 5 min exploring a $60 \times 60 \mathrm{~cm}$ sample arena enriched with five objects, followed by a $20 \mathrm{~min}$ rest in the home cage. Six old and six adult animals assigned to the $A A$ condition were then returned to the sample arena for an additional 5 min of exploration. Six old and six adult animals assigned to the $A B$ condition were instead placed in a different arena within a different testing room for an additional $5 \mathrm{~min}$ of exploration. The arenas were the same size and shape, but the walls differed in color and material. The testing rooms contained distal visual cues that were distinct from each other.

the animal colony room to rest for $20 \mathrm{~min}$. After the rest episode, the rats were either placed back into area A to explore the same five objects for another 5 min (AA) or transported to arena $\mathrm{B}$, which was located in a different room $(\mathrm{AB})$, to explore the same five objects. Halfway through the exploration time, if the rat had not explored each object, it was repositioned adjacent to the unexplored object to encourage exploration. This procedure was done in three old rats (one $\mathrm{AA}$, two $\mathrm{AB}$ ) and two adult rats (one $\mathrm{AA}$, one $\mathrm{AB}$ ), and the data obtained from these animals did not constitute outliers.

The areas were cleaned with a $70 \%$ ethanol solution after each rat had completed the behavior, and exploration was recorded by an overhead video recorder for offline analysis of exploration times. Exploration time was considered to be the periods when the rat was directing its nose toward an object within $2 \mathrm{~cm}$. Other behaviors, such as rearing or climbing on objects, were not considered to be exploration. All rats were transported between the testing arena and colony room in a covered towel-lined pot to reduce exposure to extraneous stimuli.

After completing epoch 2, each rat was anesthetized in a bell jar saturated with isoflurane and killed by decapitation. The brains were immediately removed, hemisected, and flash frozen in isopentane submerged in a dry ice and ethanol slurry. Additionally, six adult and six old rats were killed directly from the home cage without undergoing any exploration to serve as a negative control [caged control (CC)], and six adult and five old rats underwent maximal electroconvulsive shock (MECS) $30 \mathrm{~min}$ before decapitation to induce maximal Arc expression. The tissue was then grouped and mounted in optimal cutting temperature medium (VWR Scientific) such that one brain hemisphere from each combination of behavioral conditions and age group was present in a $2 \times 4$ grid in each of six blocks. The blocks were frozen sectioned into $20-\mu \mathrm{m}$-thick sections that were mounted on Superfrost Plus slides (VWR Scientific).

Fluorescence in situ hybridization. Fluorescence in situ hybridization (FISH) was performed for the immediate-early gene Arc as described previously (Guzowski et al., 2001). To summarize, digoxigenin-labeled 


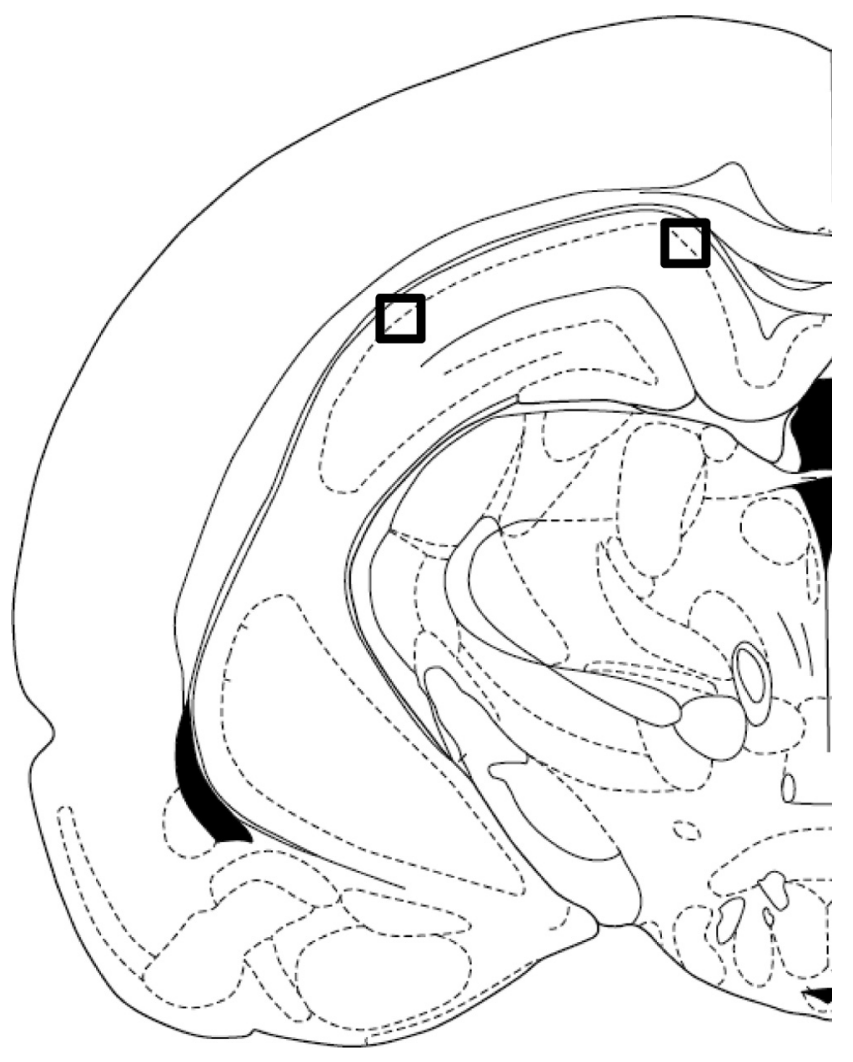

Figure 2. Proximal versus distal CA1. Diagram of the hippocampus showing where confocal images were taken after catFISH was performed on the tissue. Confocal images were taken in distal CA1 (black square on the right) and proximal CA1 (black square on the left), midway along the dorsoventral axis of the hippocampus. Distal CA1 was distinguished from the subiculum based on the decrease in cell body dispersion and the obvious increase in neuron density in the CA1 stratum pyramidale (Amaral and Witter, 1995). Proximal CA1 was differentiated from CA2 based on increased density of Arc staining in proximal CA1 relative to CA2. Specifically, CA2 shows a limited proportion of neurons that are positive for Arc because of the reduced plasticity within this subregion of the hippocampus (Zhao et al., 2007).

riboprobe was generated from a plasmid containing $3.0 \mathrm{~kb}$ Arc cDNA using a commercial transcription kit and RNA labeling mix (Ambion). The digoxigenin-labeled Arc antisense riboprobe was hybridized with the tissue overnight. The Arc riboprobe was then detected with anti-digoxigenin-HRP conjugate (Roche Applied Science) and visualized using a cyanine-3 (Cy3 Direct FISH; PerkinElmer Life Sciences) or SuperGlo Red (Fluorescent Solutions) substrate kit. Nuclei were counterstained with Sytox-green (Invitrogen) so that nuclear Arc and cytoplasmic Arc could be differentiated.

Image acquisition and analysis. Distal and proximal CA1 images were taken on a Carl Zeiss LSM 510 Meta-NLO Confocal microscope. Figure 2 shows where the images were taken along the transverse axis of CA1. Distal CA1 was distinguished from the subiculum based on the decrease in cell body dispersion and the obvious increase in neuron density in the CA1 stratum pyramidale (Amaral and Witter, 1995). Proximal CA1 was differentiated from CA2 based on the increased density of Arc staining. Specifically, CA2 shows a limited proportion of neurons that are positive for $\operatorname{Arc}$ because of the reduced plasticity within this subregion of the hippocampus (Zhao et al., 2007). In addition to using decreased Arc expression to delineate the border between proximal CA1 and CA2, we took images well away from our estimate of the border of CA2 to ensure that we were well within the CA1 boundary. The detector gain and amplifier offset settings were adjusted for a slide to reproduce the Arc expression that was seen with the eyepiece. These settings remained constant for all image stacks acquired for the slide, except in the case of uneven staining, in which settings were optimized to reproduce what was seen with the eyepiece for each image stack. To detect nuclear counterstain, detector gain and amplifier offset for a second channel were opti- mized so that non-neuronal cells, characterized by small size and bright, uniformly stained nuclei, appeared completely saturated and easily distinguishable from large, sparsely stained nuclei. These large, sparsely stained nuclei have been shown to stain with either $\alpha$ calcium/ calmodulin-dependent protein kinase II (CAMKII) or GAD65/67 in double-labeled FISH experiments, identifying them as either pyramidal neurons or interneurons, respectively (Vazdarjanova et al., 2006). In CA1, Arc mRNA is expressed in CAMKII-positive neurons but not in GAD65/67 neurons. The small, uniformly stained cells were putative glia cells and were excluded from the study, and these cells do not express Arc under standard behavioral conditions (Vazdarjanova et al., 2006). Image stacks made up of $1-\mu \mathrm{m}$-thick optical sections were collected from both proximal CA1 and distal CA1 in the middle hippocampus, approximately -4.16 to $-5.3 \mathrm{~mm}$ from bregma, from three slides per rat. All images were collected with a $40 \times$ oil-immersion objective.

Images were analyzed offline using MetaMorph (Universal Imaging Corporation) and NIH ImageJ imaging software. Only the neuronal nuclei that were present in the median $20 \%$ of each image stack were included in the analysis. This was done so that sampling errors attributable to partial cells would be minimized. Nuclei that were included in the analysis were selected with the Arc channel turned off so that Arc expression would not bias the cells that were included. Cells were classified as negative for Arc expression, positive for cytoplasmic Arc only (indicating activity during epoch 1 of object exploration), positive for nuclear Arc only (indicating activity during epoch 2 of object exploration), or positive for both cytoplasmic and nuclear Arc (indicating activity during both epochs of object exploration) by experimenters blind to the experimental conditions and animal age from which the image stack was obtained. Images from the different age groups and behavioral conditions were equally distributed among counters to account for any variability between counters. A neuron was considered positive for cytoplasmic Arc if the signal was present within the 3 pixels adjacent to the nucleus on at least three sections of the optical stack and surrounded at least $\sim 25 \%$ of the nucleus on at least one optical section. A neuron was considered positive for nuclear Arc if the signal was present within the nucleus on at least three sections of the optical stack and reached a brightness value of 255 on at least one section. Figure 3 shows a section of tissue with two of the three possible staining profiles indicated with arrows. Cell nuclei are shown in blue and Arc mRNA is shown in red. Cytoplasmic Arc (yellow arrow) indicates neurons active during behavioral epoch 1 only (25-30 min before the animals were killed). Intranuclear Arc foci (green arrow) indicate principal cell activity during behavioral epoch $2(\sim 2-5 \mathrm{~min}$ before the animals were killed). Neurons containing both nuclear and cytoplasmic Arc were active during both of the behavioral epochs. Figure 4 shows examples of tissue from animals assigned to the MECS condition, the CC condition, and a behavioral condition.

Calculation of catFISH similarity scores. A similarity score was calculated to condense the cell staining data resulting from two behavioral epochs into a single numerical value that can be compared across brain regions and experimental conditions (Vazdarjanova and Guzowski, 2004). A similarity score of 1 represents a single population of neurons that was active during both epochs of exploration, whereas a score of 0 indicates that a statistically independent population of neurons was active during each epoch. The similarity score was calculated as follows: (1) epoch 1 active cells (EI) = fraction of cytoplasmic Arc-positive cells + fraction of cytoplasmic and nuclear Arc-positive cells; (2) epoch 2 active cells (E2) $=$ fraction of nuclear Arc-positive cells + fraction of cytoplasmic and nuclear $A r c$-positive cells; (3) p(E1E2) = epoch 1 active cells $\times$ epoch 2 active cell fraction (this represents the probability of cells being active in both epochs, assuming that the two epochs activated statistically independent neuronal ensembles); (4) diff(E1E2) = fraction of cytoplasmic and nuclear Arc-positive cells - p(E1E2) (this is a measure of the deviation from the independence hypothesis); (5) least epoch $=$ the smaller of the ensembles activated by epoch 1 or epoch 2 ; and (6) similarity score $=\operatorname{diff}(\mathrm{E} 1 \mathrm{E} 2) /($ least epoch $-\mathrm{p}(\mathrm{E} 1 \mathrm{E} 2))$ [this normalizes the diff(E1E2) fraction to a perfect A/A condition; a perfect A/A is 1 , and a perfect A/B is zero] (Vazdarjanova and Guzowski, 2004).

Calculation of path overlap scores. The degree to which rats visited the same spatial locations between epochs 1 and 2 of behavior was quantified 


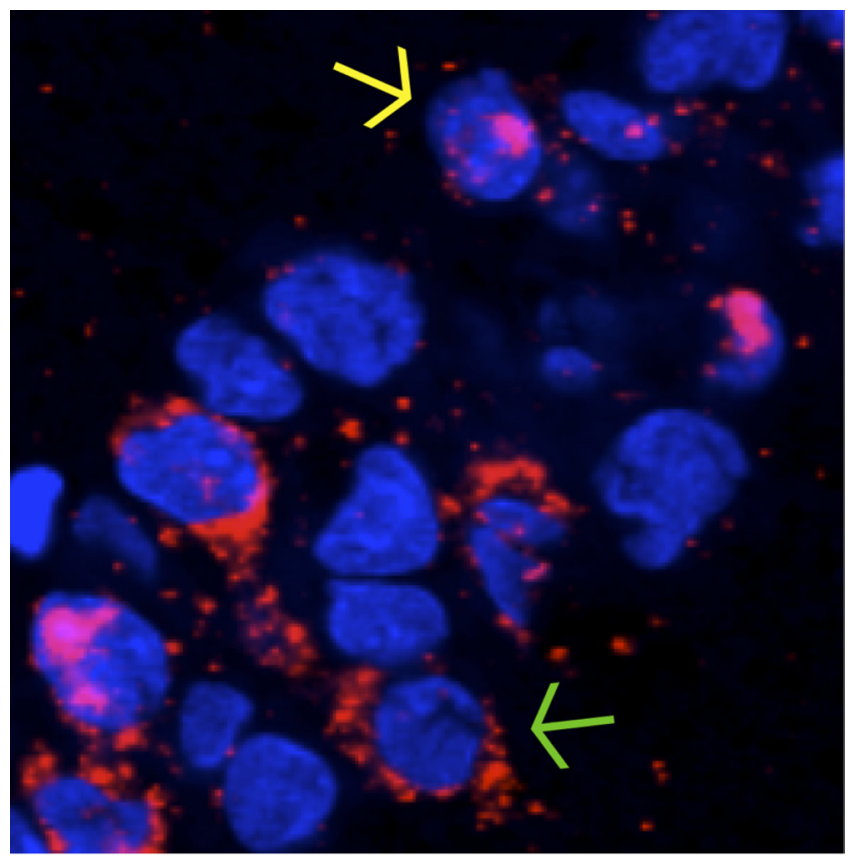

Figure 3. The subcellular distribution of Arc. An example of Arc expression (red) in CA1 neurons as visualized by catFISH after two exposures to an environment. Neuron nuclei are represented in blue. Nuclear Arc foci (yellow arrow) indicate that a neuron was active during the second epoch of exploration. (ytoplasmic Arc (green arrow) indicates neuron activity during the first round of exposure. The presence of both cytoplasmic and nuclear Arc expression indicates that a neuron was active during both exploratory epochs.

by calculating a value referred to here as the "path overlap score." This value was calculated by first dividing the exploratory arena into a $3 \times 3$ grid and tabulating the number of visits each rat made to the nine cells during each epoch of exploration. A visit was counted when the rat's front paws crossed the threshold into a new cell. These values were incorporated into two different nine-dimensional vectors, one vector for epoch 1 and another for epoch 2. Then, the dot product of these two vectors was calculated and normalized to a scale from 0 to 1 by dividing the product of the vectors by the product of the magnitude of each vector. The formula for the calculation of this value is as follows:

$$
\begin{gathered}
e 1(c 1, c 2, c 3, c 4, c 5, c 6, c 7, c 8, c 9) \\
\times \quad e 2(c 1, c 2, c 3, c 4, c 5, c 6, c 7, c 8, c 9) \\
\hline|e 1||e 2|
\end{gathered}
$$

where $e$ represents the epoch of exploration, and $c$ represents the number of visits to each of the nine cells.

Statistical analyses. For all statistical tests, the mean values of each variable were calculated for individual rats, and that value served as the $n$ rather than the number of images or slides. This was done to avoid inflating statistical power with a large number of observations. The $\alpha$ level for each statistical test was set to 0.05 .

\section{Results}

\section{Morris swim task}

To assess the spatial memory and visual acuity of the rats used in this experiment, the 12 adult and 12 old rats that participated in the object-exploration procedures performed the spatial and visually cued Morris swim task (Morris, 1984). Figure 5 shows the CIPL values (Gallagher et al., 1993) averaged for the adult and old rats during the spatial and visually cued trials of this task. The CIPL quantifies the cumulative search error independent of start location and swimming speed.

During the $4 \mathrm{~d}$ of spatial trials, adult and old rats were required to find the location of a hidden escape platform underneath the surface of opaque water. There was a significant main effect of day on CIPL scores $\left(F_{(3,60)}=13.79, p<0.001\right.$, repeated-measures ANOVA). Planned simple contrasts revealed that performance on day 4 was significantly better than on all proceeding days $(p<$ 0.001 for all comparisons), demonstrating that all rats showed some learning of the location of the platform over time. There was also a significant difference in the performance of adult rats versus old rats, with old rats showing significantly longer paths to reach the platform, independent of differences in swimming velocity $\left(F_{(1,20)}=7.37, p<0.01\right)$. This suggests that the adult rats used in this study had, on average, better spatial memory than did the old group. Importantly, there was not a significant difference in the performance of the rats assigned to the $A A$ versus $A B$ behavioral conditions $\left(F_{(1,20)}=0.01, p>0.91\right)$, as well as no significant interaction between age and behavioral group $\left(F_{(1,20)}=0.07\right.$, $p>0.80)$.

During the visually cued trials of the Morris swim task, there was no significant difference between the performances of adult versus old rats $\left(F_{(1,20)}=1.14, p>0.29\right)$, indicating that the age groups had comparable visual acuity. There was also no significant difference in the performance of rats assigned to each behavioral condition $\left(F_{(1,20)}=0.40, p>0.53\right)$. Finally, the mean CIPL values on day 1 and day 2 were not significantly different $\left(F_{(1,20)}=2.69, p>0.12\right.$, repeated-measures ANOVA).

\section{Object exploration, threshold crossings, and path overlap score}

To investigate the population activity overlap in distal versus proximal CA1 neurons after two epochs of object exploration, the adult (7-9 months old) and old (24-26 months old) rats underwent two 5 min exposures to objects separated by $20 \mathrm{~min}$ in which the testing environment either changed (condition $\mathrm{AB}$ ) or remained consistent (condition AA) between exposures (Fig. 1, $n=$ 12 for each group). All rats explored each object at least once in both the first and second epochs of behavior. The total amount of time spent exploring all objects during epoch 1 and epoch 2 for the adult and old rats is shown in Figure 6A. The rats spent significantly more time exploring the five objects during epoch 1 , when they were novel, compared with epoch $2\left(F_{(1,20)}=24.77\right.$, $p<0.001$, repeated-measures ANOVA). There was also a significant main effect of age on object-exploration time (repeatedmeasures ANOVA, $\left.F_{(1,20)}=6.68, p<0.02\right)$, with the old rats spending less time exploring the objects. The reduction in exploration time between epochs did not interact significantly with age group $\left(F_{(1,20)}=0.52, p=0.48\right.$, repeated-measures ANOVA) or behavioral condition $\left(F_{(1,20)}=0.12, p=0.73\right.$, repeated-measures ANOVA). These data indicate that both the adult and old rats behaved as if they recognized that the objects were familiar during the second epoch of behavior even when the environment changed (Ennaceur and Delacour, 1988).

Although the rats spent less time exploring objects during epoch 2 relative to the first epoch of behavior, when the arena was divided into nine cells and each rat's path of exploration was traced to measure the total number of threshold crossings, there was no significant effect of epoch on threshold crossings $\left(F_{(1,20)}=\right.$ $0.93, p=0.35$, repeated-measures ANOVA). This suggests that the reduction in object exploration between epochs was not associated with lower levels of environmental exploration. Moreover, the number of threshold crossings did not significantly differ between behavioral conditions (AA vs $\mathrm{AB} ; F_{(1,20)}=0.10$, $p=0.76$ ). The observation that the rats assigned to the $\mathrm{AB}$ condition did not exhibit an increase in the number of threshold crossings during epoch 2 relative to those in the AA condition can 

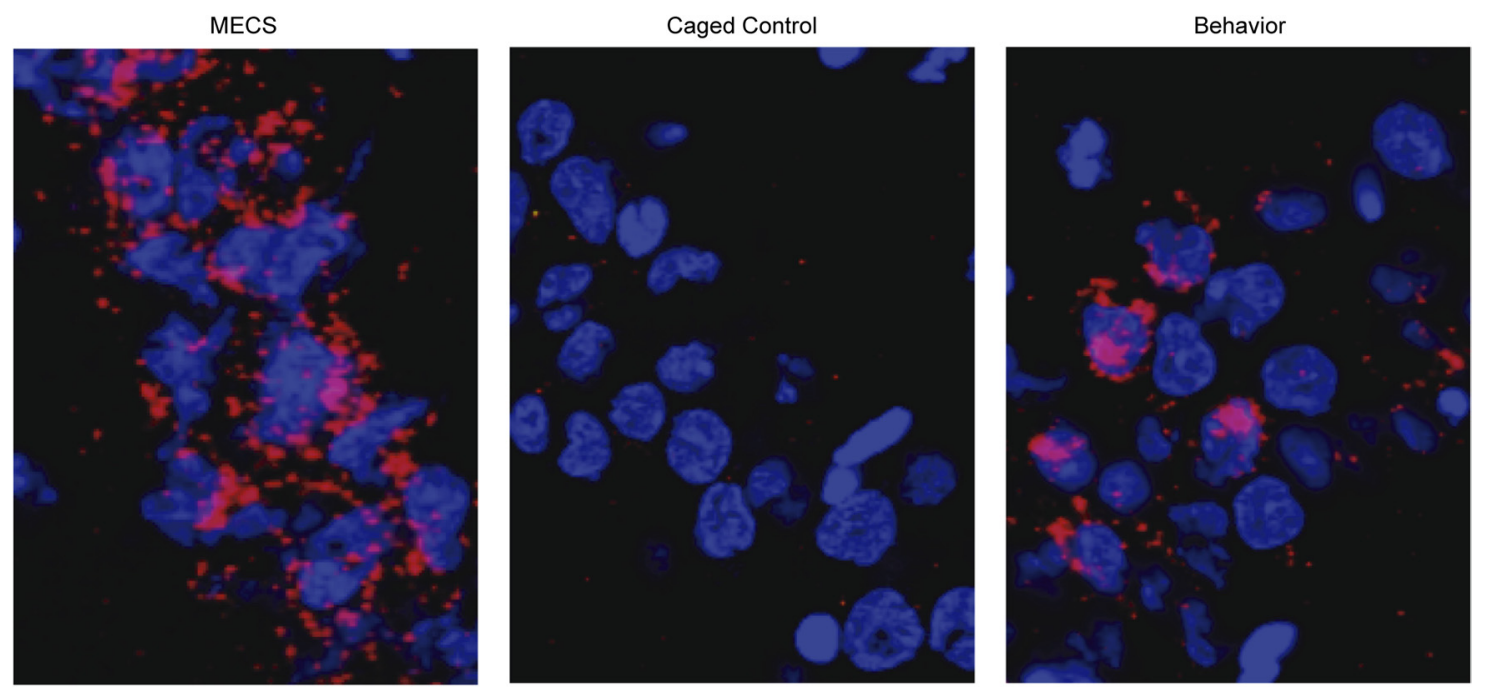

Figure 4. Arc expression in the different conditions. Examples of Arc expression as visualized by catFISH in CA1 neurons from animals assigned to the MECS condition (left), the CC condition (middle), and a behavioral condition (right). Neuronal nuclei are shown in blue, and Arc mRNA is shown in red.

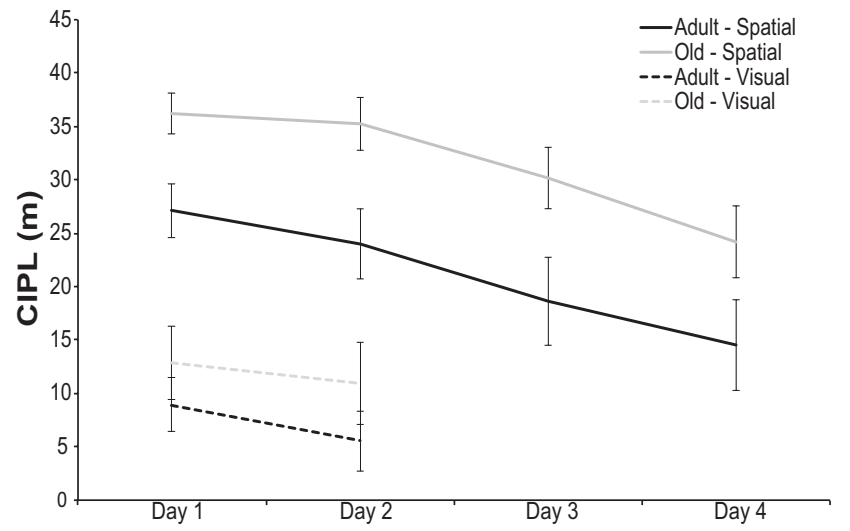

Figure 5. Morris swim task performance. The CIPL for adult and old rats during spatial (solid lines) and visual (dashed lines) trials is shown on the $y$-axis as a function of training day ( $x$-axis). During the spatial trials, there was a significant main effect of day on CIPL scores (repeatedmeasures ANOVA, $\left.F_{(3,60)}=13.79, p<0.001\right)$. There was also a significant difference in CIPL scores between age groups (ANOVA, $\left.F_{(1,20)}=7.37, p<0.01\right)$. During the visual trials, there was no significant difference in the performance between age groups (ANOVA, $F_{(1,20)}=1.14$, $p>0.29$ ) on day 1 and day 2 (repeated-measures ANOVA, $F_{(1,20)}=2.69, p>0.12$ ). Error bars indicate \pm 1 SEM.

be explained by the fact that the animals had previous exposure during the habituation phase to both environments A and B. On average, adult rats showed significantly more threshold crossings than did old rats $\left(F_{(1,20)}=13.73, p<0.01\right)$, indicating reduced levels of motor output in the old rats. However, the difference in voluntary exploration between adult and old rats did not significantly affect the proportion of cells positive for Arc mRNA (see below). The average number of threshold crossings for each group during each of the two epochs of exploration is shown in Figure $6 B$.

The mean path overlap score for adult (black) and old (gray) rats is shown in Figure 7. There was a significant effect of path overlap between epochs 1 and 2 in both age group (adult vs old; $F_{(1,20)}=11.13, p<0.01$ ) and behavioral conditions (AA vs AB; $\left.F_{(1,20)}=7.74, p<0.05\right)$. Specifically, there was less betweenepoch path overlap in old rats relative to the adult animals. Moreover, the AA condition had significantly more path overlap compared with the $\mathrm{AB}$ condition, but there was no significant inter- action between age group and behavioral condition $\left(F_{(1,20)}=0.02\right.$, $p=0.90$ ). These data suggest that old rats were less likely to occupy the same spatial locations between epochs of exploration. The influence that this behavioral variable has on the population overlap of CA1 neural ensembles will be considered further with respect to the similarity score (see below).

\section{Cell numbers}

When the total numbers of pyramidal cells included in the current analysis were compared across age group and region of CA1, there was no significant effect of age group on the number of active neurons (Table 1$)\left(F_{(1,77)}=0.92, p=0.34\right)$. This is consistent with previous data showing no loss of CA1 neurons in advanced age (Rapp and Gallagher, 1996). The total number of cells analyzed for each behavioral condition (AA vs $\mathrm{AB}$ ) was also not significantly different $\left(F_{(3,77)}=1.00, p=0.40\right)$. Additionally, there was no significant interaction between condition and age group $\left(F_{(3,77)}=0.99, p=0.40\right)$. However, there was a significant difference in the number of cells counted in the distal compared with the proximal region of CA1 (ANOVA, $F_{(1,77)}=4.31, p<$ $0.05)$. This difference is likely attributable to the different orientation of the cell body layer to the field of view of the confocal microscope in the distal versus proximal regions of CA1, such that a longer extent of the cell body layer was included for distal CA1 because it crossed the field of view at a steeper angle compared with proximal CA1. The number of cells within each region did not significantly interact with any other variable $(p>0.1$ for all comparisons). Together, these data indicate that comparisons between conditions and age groups were not confounded by differences in cell number.

\section{Proportion of cells expressing Arc}

The proportion of cells positive for Arc mRNA for distal and proximal CA1 in the different age groups and behavioral conditions are shown in Figure 8. Behavioral condition (AA, AB, MECS, and CC) had a significant effect on the portion of cells expressing $\operatorname{Arc}\left(F_{(3,39)}=60.15, p<0.001\right.$, repeated-measures ANOVA). Specifically, rats in the MECS condition had a significantly higher percentage of $A r c$-positive CA1 pyramidal cells than did the rats in the other three conditions (Tukey's HSD test, $p<$ 0.001 for all comparisons). Moreover, rats in the CC condition 

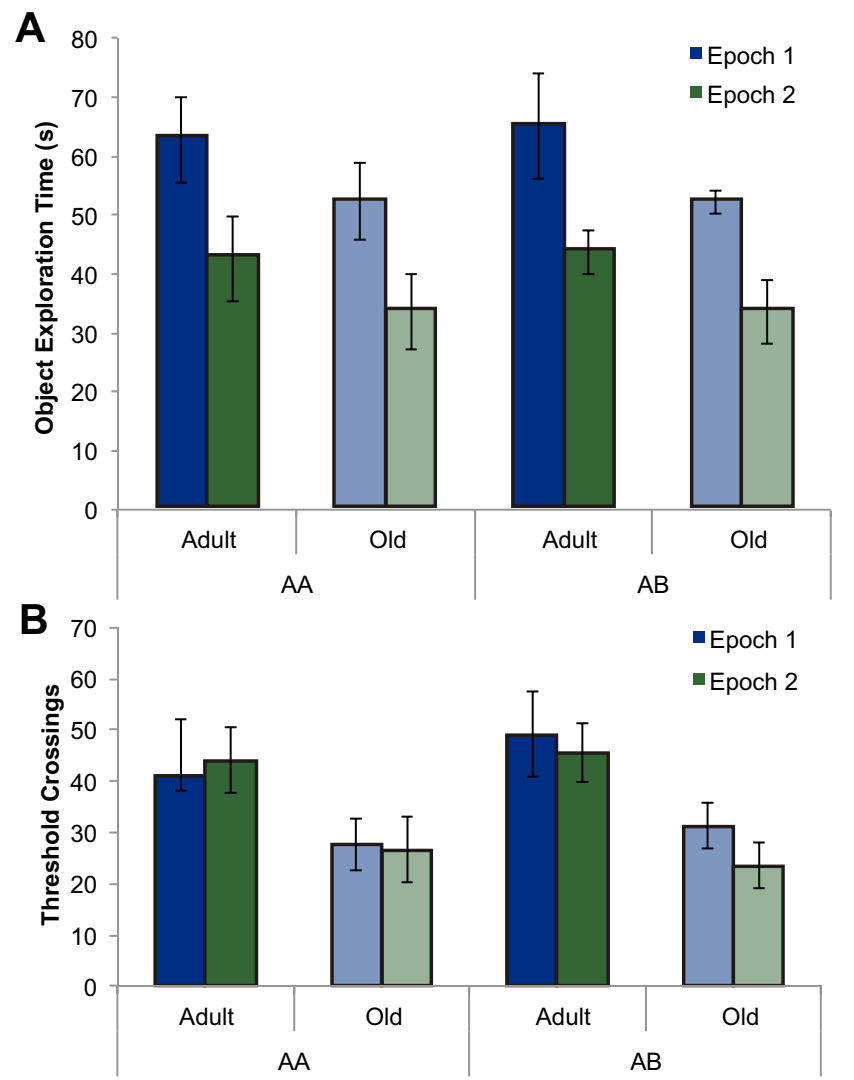

Figure 6. Behavior during epochs of exploration. $A$, Object exploration time for adult and old rats during the two epochs of exploration. Object exploration was counted when a rat directed its nose toward an object while within $2 \mathrm{~cm}$ of it. Rats explored the five objects for significantly more time during behavioral epoch 1 relative to epoch 2 (repeated-measures ANOVA, $F_{(1,20)}=$ $24.8, p<0.001$ ), indicating that the rats recognized the objects as familiar during epoch 2. There was no significant difference in exploration time between rats assigned to the AA condition versus $A B$ condition ( $A N O V A, F_{(1,20)}=0.13, p>0.72$ ). However, there was a significant main effect of age (repeated-measures ANOVA, $F_{(1,20)}=6.68, p<0.02$ ), with the old rats spending less time exploring the objects. Error bars indicate \pm 1 SEM. $\boldsymbol{B}$, Number of threshold crossings for adult and old rats during the two epochs of exploration. There was no significant effect of epoch on threshold crossings $\left(F_{(1,20)}=0.93, p=0.35\right.$, repeated-measures ANOVA). Moreover, the number of threshold crossings did not significantly differ between behavioral conditions ( $A A$ vs $\left.A B ; F_{(1,20)}=0.10, p=0.76\right)$. On average, adult rats made a significantly greater number of threshold crossings than did old rats $\left(F_{(1,20)}=13.73, p<0.01\right)$. Error bars indicate \pm 1 SEM.

had significantly lower normalized numbers of $A r c$-positive neurons in $\mathrm{CA} 1$ relative to the rats in both the $\mathrm{AA}$ and $\mathrm{AB}$ conditions (Tukey's HSD test, $p<0.001$ for all comparisons). Although there was a difference in the amount of time spent exploring objects and the number of threshold crossings between adult and old rats, there were no significant age effects on the proportion of cells expressing Arc (repeated-measures ANOVA, $F_{(1,39)}=0.13$, $p>0.73)$. This is consistent with previous work that has reported no reduction in the numbers of CA1 neurons that express $A r c$ in old animals (Small et al., 2004; Penner et al., 2011). There was also not a significant interaction effect of age group and behavioral condition ( $p>0.08$ for all comparisons).

The subcellular distribution (foci only, cytoplasm only, or both) of $A r c$ mRNA is shown for the positive and negative control data in Figure 9. There was no significant effect of age group (adult vs old; $F_{(2,76)}=0.40, p=0.67$ ) or region (proximal vs distal; $F_{(2,76)}=0.09$, $p=0.91$ ) on Arc distribution for the two control conditions. However, there was a difference in the proportion of cells with Arc foci only, cytoplasm only, or both $\left(F_{(2,76)}=183.93, p<0.001\right)$, and this

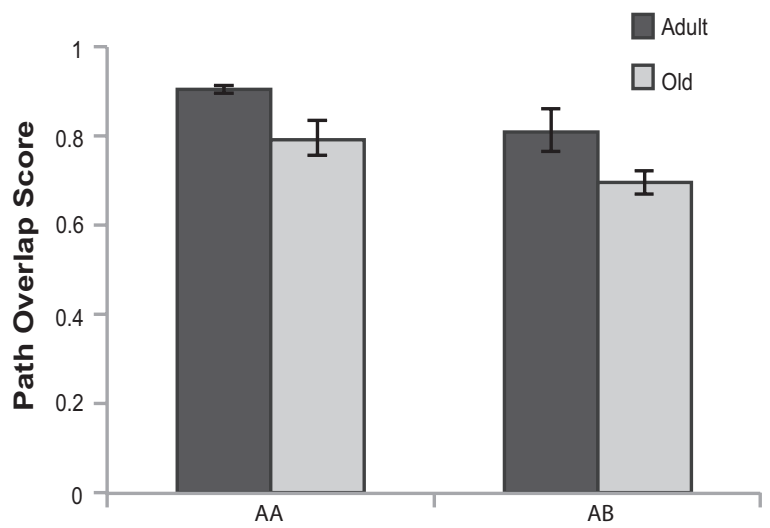

Figure 7. Path overlap score between epochs of exploration. The $y$-axis shows the path overlap between epochs 1 and 2 for the AA and AB behavioral conditions ( $x$-axis) in adult (dark gray) and old (light gray) rats. There was a significant effect on path overlap of age (adult vs old; $\left.F_{(1,20)}=11.13, p<0.01\right)$ and behavioral condition (AA vs $\left.A B ; F_{(1,20)}=7.74, p<0.05\right)$. Error bars indicate $\pm 1 \mathrm{SEM}$.

Table 1. Total number of CA1 neurons counted in distal and proximal CA1 in adult and old rats

\begin{tabular}{|c|c|c|}
\hline & \multicolumn{2}{|l|}{ Mean \pm SEM } \\
\hline & Distal CA1 & Proximal CA1 \\
\hline \multicolumn{3}{|l|}{$A A$} \\
\hline Adult & $61.28 \pm 2.99$ & $66.11 \pm 3.36$ \\
\hline Old & $62.72 \pm 2.25$ & $70.83 \pm 3.84$ \\
\hline \multicolumn{3}{|l|}{$A B$} \\
\hline Adult & $62.22 \pm 2.15$ & $68.47 \pm 4.40$ \\
\hline Old & $55.06 \pm 2.83$ & $66.33 \pm 3.95$ \\
\hline \multicolumn{3}{|l|}{ MECS } \\
\hline Adult & $66.28 \pm 2.89$ & $72.89 \pm 2.79$ \\
\hline Old & $62.87 \pm 3.76$ & $63.40 \pm 3.86$ \\
\hline \multicolumn{3}{|l|}{$C C$} \\
\hline Adult & $60.67 \pm 2.46$ & $62.28 \pm 2.02$ \\
\hline Old & $61.61 \pm 3.51$ & $60.72 \pm 2.98$ \\
\hline \multicolumn{3}{|l|}{ Total } \\
\hline Adult & $62.61 \pm 1.32$ & $67.44 \pm 1.65$ \\
\hline Old & $60.56 \pm 1.56$ & $65.32 \pm 1.85$ \\
\hline
\end{tabular}

difference in the subcellular distribution of Arc varied significantly by control condition (MECS vs CC; $F_{(2,76)}=186.59, p<0.001$ ). In particular, for the MECS condition, there were significantly more neurons with Arc in the cytoplasm only ( $p<0.01$, Tukey's HSD test). This is consistent with previous experiments that administered MECS 30 min before the animals were killed (Guzowski et al., 1999).

\section{Distribution of Arc mRNA after two epochs of exploration}

Figure 10 shows the distribution of Arc mRNA (foci only, cytoplasm only, or both) in the $\mathrm{AA}$ and $\mathrm{AB}$ conditions for the proximal versus distal CA1 region of adult versus old rats. Overall, the distal portion of CA1 had a significantly higher proportion of cells that were positive for both cytoplasmic and nuclear Arc signal relative to the proximal region of CA1 (ANOVA, $F_{(1,40)}=$ 6.62, $p<0.02)$. This indicates that, in distal CA1, it was more likely for a neuron to show activity during both epochs of behavior compared with proximal CA1. Additionally, the adult rats showed a significantly higher proportion of principal cells that were positive for both nuclear and cytoplasmic Arc signal compared with the old rats $\left(F_{(1,40)}=6.90, p<0.02\right)$. Possible explanations of the reduced overlap in the active neural ensemble between epochs in old rats will be explored further in the next section. When data from the different regions of CA1 and age groups were analyzed together, there was no significant effect of condition 


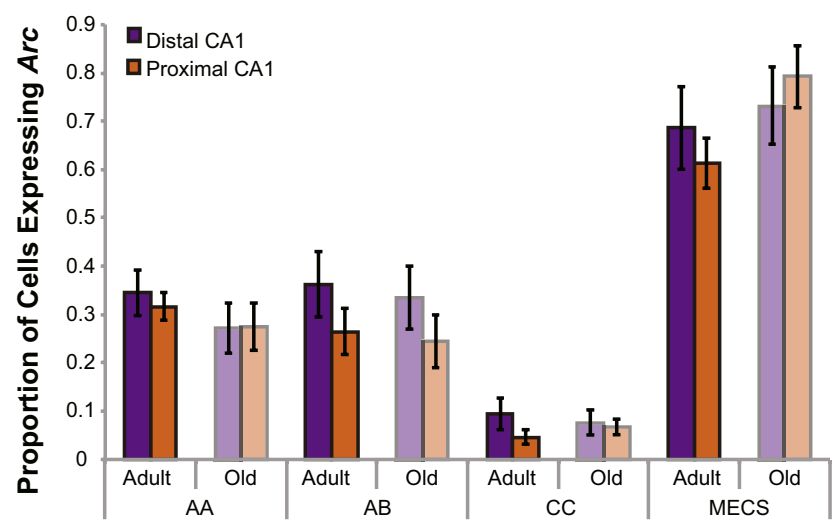

Figure 8. Proportion of CA1 cells that expressed Arc across the different age groups and behavioral conditions. The $y$-axis shows the proportion of distal (purple) and proximal (orange) CA1 cells that were positive for Arc mRNA (in any cellular compartment) as a function of age group and experimental condition (AA, $A B, C C$, or MECS). Behavioral condition (but not age) significantly affected the proportion of cells that expressed Arc (repeated-measures ANOVA, $\left.F_{(3,39)}=60.15, p<0.001\right)$. Specifically, rats in the MECS condition had a significantly higher percentage of Arc-positive CA1 pyramidal cells than did the rats in the other three conditions (Tukey's HSD test, $p<0.001$ for all comparisons). Moreover, rats in the (C condition had significantly lower normalized numbers of Arc-positive neurons in CA1 relative to the rats in both the $A A$ and $A B$ conditions (Tukey's HSD test, $p<0.001$ for all comparisons). Error bars indicate \pm 1 SEM.

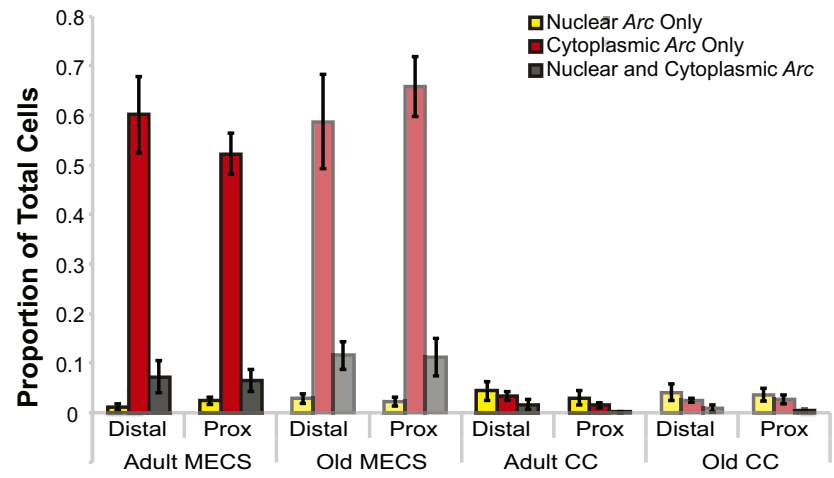

Figure 9. Arc distribution in the negative and positive control conditions. Arc expression profiles by age for rats killed $30 \mathrm{~min}$ after undergoing MECS or killed directly from the home cage (CC). There was no significant effect of age group (adult vs old; $F_{(2,76)}=0.40, p=0.67$ ) or region (proximal vs distal; $F_{(2,76)}=0.09, p=0.91$ ) on Arc distribution for the two control conditions. However, there was a difference in the proportion of cells with Arc foci only, cytoplasm only, or both $\left(F_{(2,76)}=183.93, p<0.001\right)$, and this difference in the subcellular distribution of Arc varied significantly by control condition (MECS vs CC; $F_{(2,76)}=186.59, p<0.001$ ). In particular, for the MECS condition (as expected at the 30 min time point), there were significantly more neurons with Arc in the cytoplasm only ( $p<0.01$, Tukey's HSD test). Error bars indicate \pm 1 SEM

(AA vs $\mathrm{AB}$ ) on the proportion of cells showing both cytoplasmic and nuclear Arc signal (ANOVA, $F_{(1,40)}=3.17, p=0.08$ ).

\section{catFISH similarity scores}

A calculation of similarity scores (Vazdarjanova and Guzowski, 2004) can also be used to characterize the subcellular distribution of $\operatorname{Arc}$ (foci only, cytoplasm only, or both) with a single value that provides a quantitative measure of the population overlap of cell activity patterns between the two different epochs of behavior. The similarity score provides a better means for comparing overlapping activity patterns across brain regions and age groups because it controls for differences in cell number and overall proportion of positive cells. A low similarity score is consistent

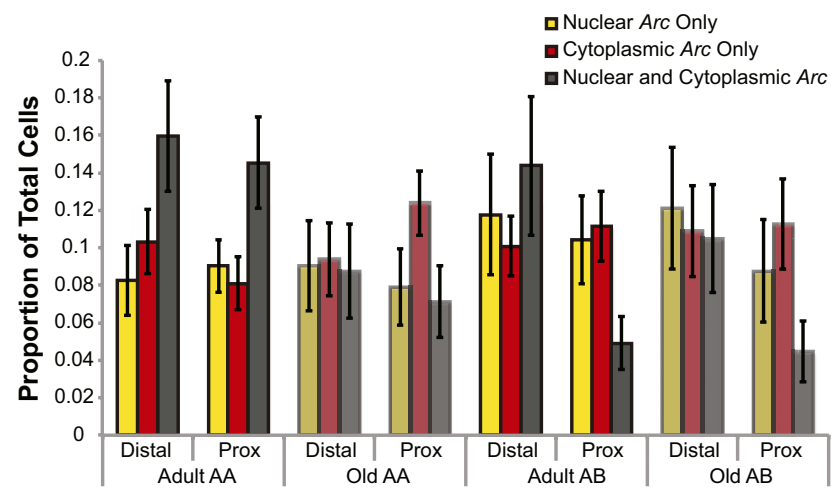

Figure 10. Arc distribution in the AA and AB behavioral conditions. Arc expression profiles by age for rats either exposed twice to the same set of five objects within the same environment $(A A)$ or exposed twice to the same objects within two different environments ( $A B)$. Overall, the distal portion of CA1 had a significantly higher proportion of cells that were positive for both cytoplasmic and nuclear Arc signal, indicating that these cells were active during both epochs of behavior relative to the proximal region of $C A 1\left(F_{(1,40)}=6.62, p<0.02\right)$. Additionally, the adult rats showed a significantly higher proportion of principal cells that were positive for both nuclear and cytoplasmic Arc signal compared with the old rats $\left(F_{(1,40)}=6.90, p<0.02\right)$, suggesting that neural ensembles in old CA1 exhibited a greater degree of remapping. When data from the different regions of $C A 1$ and age groups were analyzed together, there was no significant effect of condition ( $A A$ vs $A B$ ) on the proportion of cells showing both cytoplasmic and nuclear Arc signal (ANOVA, $F_{(1,40)}=3.17, p=0.08$ ). The lack of an effect of behavioral condition on population overlap was likely attributable to the old rats showing reduced overlap in the AA condition. Error bars indicate \pm 1 SEM.

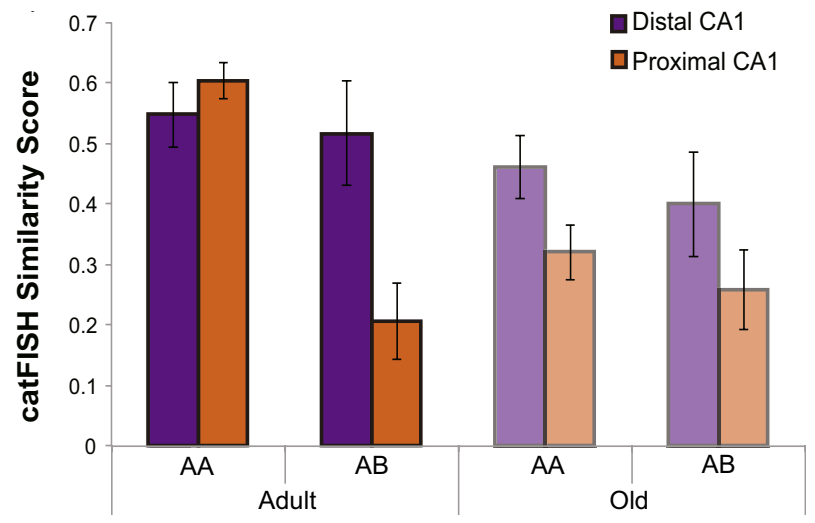

Figure 11. Ensemble overlap in distal versus proximal CA1. catFISH similarity scores for rats either exposed twice to the same set of five objects within the same environment (AA) or exposed twice to the same objects within two different environments $(A B)$. In adult rats, there were significant differences in catFISH similarity scores for both distal and proximal CA1 between the $A A$ and $A B$ conditions $\left(F_{(1,20)}=21.3, p<0.01\right.$, repeated-measures ANOVA), with adult rats showing a significantly greater degree of remapping during the $A B$ condition compared with the $A A$ condition. Interestingly, old rats showed no significant difference between the catFISH similarity scores for the $A A$ and $A B$ conditions $\left(F_{(1,20)}=0.68, p=0.42\right.$, repeatedmeasures ANOVA). Additionally, in adult rats, the difference in catFISH similarity score between the $A A$ and $A B$ condition was significantly larger in proximal $C A 1$ than distal $C A 1\left(F_{(1,10)}=\right.$ $17.41, p<0.01$, repeated-measures ANOVA). Error bars indicate \pm 1 SEM.

with a remapping of CA1 neural ensembles, whereas a high score suggests that the same population of cells was active during both epochs of behavior. Figure $11 A$ shows that mean similarity scores for proximal and distal CA1 for the different age groups and behavioral conditions. Overall, the similarity score significantly differed between proximal and distal CA1 $\left(F_{(1,20)}=15.55, p<\right.$ 0.01 , repeated-measures ANOVA), and this difference varied significantly as a function of behavioral condition $\left(F_{(1,20)}=7.39\right.$, $p<0.05$, repeated-measures ANOVA). Specifically, post hoc 
analysis indicated that the difference in the similarity scores between distal and proximal CA1reached statistical significance for the $\mathrm{AB}$ condition ( $p<0.01$, Tukey's HSD test) but not the AA condition ( $p=0.51$, Tukey's HSD test). This suggests that the neural ensembles in proximal CA1 are more sensitive to environmental change than those in distal CA1. Interestingly, the reduced remapping in distal $\mathrm{CA} 1$ in the $\mathrm{AB}$ condition could be attributable to the fact that objects remained unchanged between epochs of behavior.

Although there was not a significant main effect of age group on similarity score $\left(F_{(1,38)}=2.90, p=0.10\right.$, repeated-measures ANOVA), there was a significant three-way interaction of behavioral condition (AA vs $\mathrm{AB}$ ), age group (adult vs old), and region of CA1 (distal vs proximal) on the similarity score. Post hoc analysis revealed that this interaction effect was attributable to the adult rats having lower similarity scores for the $\mathrm{AB}$ condition relative to the AA group ( $p<0.01$, Tukey's HSD test), but this difference in similarity scores as a function of behavior was not observed in the old rats ( $p=0.4$, Tukey's HSD test). Additionally, in adult rats, the difference in catFISH similarity score between the $\mathrm{AA}$ and $\mathrm{AB}$ conditions was significantly larger in proximal CA1 than distal CA1 ( $p<0.01$, Tukey's HSD test). In contrast, for the old rats, there were no significant effects of condition or region of CA1 on similarity score ( $p>0.1$ for all comparisons, Tukey's HSD test).

When the two different age groups were compared directly, the old rats had significantly lower similarity scores compared with the adult animals in proximal CA1 for the AA condition $(p<0.05$, Tukey's HSD test). However, for distal CA1, the similarity score for the AA condition was not significantly different between age groups ( $p=0.98$, Tukey's HSD test). There were also no significant differences between the similarity scores of adult and old rats for the $\mathrm{AB}$ condition in either proximal ( $p=$ 0.99 , Tukey's HSD test) or distal ( $p=0.89$, Tukey's HSD test) CA1. Together, these data suggest that neurons in proximal CA1 of old rats may be more likely to remap inappropriately (i.e., when a rat is returned to a familiar environment). However, differences in the behavior of adult and old rats provide an alternate explanation for the age difference in the similarity score of proximal CA1 neurons. Specifically, between epochs 1 and 2, the old rats had significantly less overlap in the exploration paths through the environment compared with the adult animals. Therefore, it is possible that the old rats did not traverse the same place fields between epochs of behavior although they remained in the same arena, and this may have contributed to the reduced similarity score.

To evaluate whether path differences between epochs contributed to reduced cell overlap, the correlation between path overlap and ensemble similarity was measured for distal and proximal CA1. Because disparities between age groups can produce erroneous correlation values, all similarity score and path overlap values were $Z$-score normalized within each age group and behavioral condition. Figure 12 shows the scatter plots of normalized similarity scores as a function of normalized path overlap for proximal and distal CA1 in the $\mathrm{AB}$ (Fig. 12A,B) and AA (Fig. 12C,D) conditions. A Bonferroni's correction was applied to compensate for calculating four correlation statistics; therefore, the $\alpha$ value was set to $p<0.013$. For the AB condition, there was no significant correlation of between-epoch path overlap and the similarity score in either distal $\left(r_{(10)}=-0.25\right.$, $p=0.44)$, or proximal $\left(r_{(10)}=-0.09, p=0.79\right)$ CA1. This indicates that, when the environment changed between epochs, variation in the spatial locations that were traversed in the arena did not affect the degree to which activity patterns overlapped between epochs of the

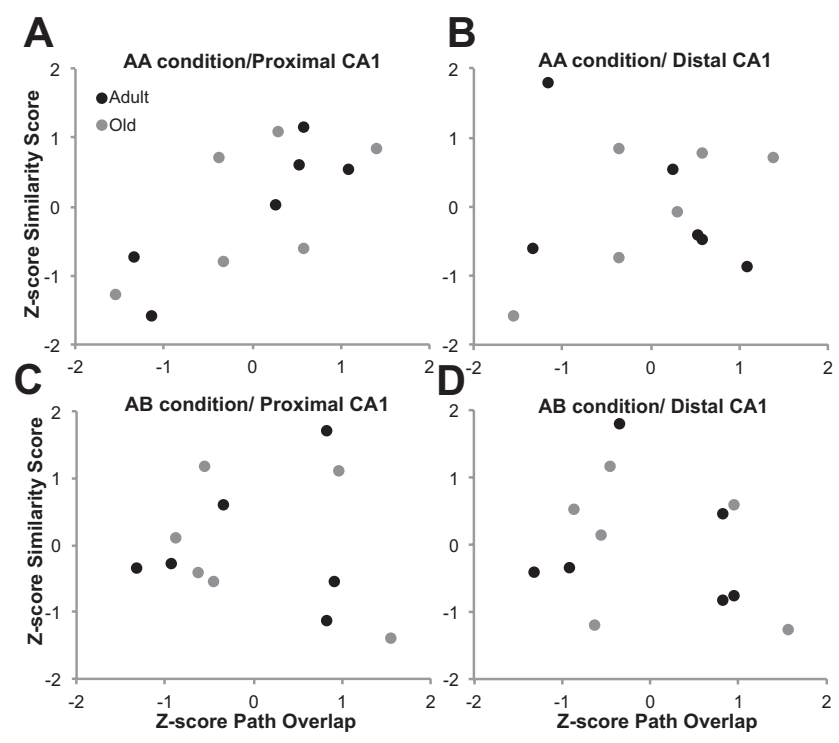

Figure 12. Normalized path overlap and ensemble similarity between epochs 1 and 2. Scatter plots of the Z-score-normalized similarity score as a function of Z-score-normalized path overlap in adult (black) and old (gray) rats for the $A A(A, B)$ and the $A B(C, D)$ conditions in distal and proximal $C A 1$. There was a significant correlation between the similarity score of the active ensembles during epochs 1 and 2 and path overlap in proximal $C A 1$ for the AA condition only ( $\boldsymbol{C}$; $\left.r_{(10)}=0.75, p<0.005\right)$. None of the other variables showed any significant correlation with path overlap ( $p>0.4$ for all comparisons).

$\mathrm{AB}$ condition. This is consistent with the idea that the active ensembles of CA1 neurons remapped when the environment changed. In contrast to the $\mathrm{AB}$ condition, during the $\mathrm{AA}$ condition, the path overlap measure was significantly correlated with the similarity score calculated from the patterns of Arc expression in proximal CA1 $\left(r_{(10)}=0.75, p<0.005\right)$ but not distal CA1 $\left(r_{(10)}=0.13, p=0.69\right)$. Thus, more path overlap between epochs of behavior occurring in the same environment suggests that the rats were more likely to traverse the same fields and therefore have higher levels of overlap in the populations of active cells. The lack of a significant correlation between the similarity score calculated from distal CA1 cells and path overlap is likely attributable to the observation that neurons in distal CA1 have more dispersed firing fields and are more likely to have multiple place fields (Henriksen et al., 2010). Thus, if a rat's path overlap is reduced between epochs, it is more likely to traverse the same distal CA1 place fields than the same proximal CA1 place fields. Importantly, the significant correlation between path overlap and similarity score in proximal CA1 for the AA condition suggests that the reduced neuron population overlap in old rats is attributable to differences in the place fields that were traversed between epochs 1 and 2 rather than inappropriate remapping. This finding can also account for the observation that the similarity scores for the AA condition were significantly different between adult and old rats in proximal but not distal CA1.

\section{Discussion}

The current findings provide strong support for the idea that CA1 pyramidal cells show distinct functional properties depending on whether their primary cortical input is from the MEC or LEC. Under the behavioral condition in which the external environment changed between two epochs of object exploration but the objects remained the same, the neuronal ensemble in proximal CA1 exhibited global remapping more often than did ensembles in distal CA1. This difference in remapping between the two CAl subregions is consistent with Henriksen et al. (2010) who observed stronger spa- 
tial modulation of proximal CA1 pyramidal cell firing compared with distal CA1 cells. The use of Arc catFISH in the current study as a cell activity marker allowed a much larger sample to be mapped across the proximodistal axis within an animal simultaneously.

One possible explanation for the observation that global remapping did not occur in distal CA1 when the spatial context changed but the salient local cues (objects) remained the same is the direct input that distal CA1 receives from object-responsive cells in both the LEC (Deshmukh and Knierim, 2011) and PRC (Burke et al., 2012c; Deshmukh et al., 2012). The stable local objects may have provided a nonspatial contextual anchor for the distal CA1 cells. In fact, these data are consistent with Burke et al. (2011a) who describe object-modulated firing of distal CA1 pyramidal cells and remapping when the position of objects on a track are shuffled or the objects are removed but the environment does not change.

These results are also interesting to consider with respect to the findings of Schmidt et al. (2012). The authors visualized Arc expression to compare population activity between distal and proximal CA1 while rats found reward in a radial arm maze using two different cognitive strategies. They report a greater degree of remapping in distal relative to proximal CA1 in response to changes in task demands in the same environment, consistent with the idea of greater sensitivity to nonspatial changes in context or nonspatial features of an episode. Because reward location was repositioned on the maze between trials, another possible interpretation of these data is that reward acts as a salient cue, and changing reward location in the testing environment prompts the remapping.

The current data should also be considered with respect to results from experiments that manipulated the spatial location of local cues on a track relative to external cues in the environment. Specifically, when the relative position of local and environmental cues is mismatched, approximately equal proportions CA1 neurons follow local cues, environmental cues, or change their firing patterns (Lee et al., 2004). Based on the current data, distal CA1 neurons might be predicted to follow local cues, whereas proximal CA1 neurons might follow environmental cues or remap. A possible explanation for the finding by Lee et al. (2004) is that the recordings were made in the intermediate and proximal zones of CA1. Thus, the recorded CA1 population may have included cells with input from MEC and LEC. Interestingly, CA3 neuron populations exhibit strong coherence under this cue mismatch paradigm, with a majority of the cells following the local cues after the rotation (Lee et al., 2004). A possible explanation for the CA3 cue mismatch results is the fact that, unlike neurons in CA1, all CA3 pyramidal cells receive direct input from the LEC (Amaral and Witter, 1995).

Another novel finding from the current experiment is that old rats did not show high ensemble overlap in distal CA1 when the environment changed and reduced overlap in proximal CA1. In fact, the degree of remapping that occurred in old animals during the $\mathrm{AB}$ condition was not significantly different between regions. One possible explanation for this age effect is altered responsiveness to local object cues. Cells in the PRC are known to be responsive to three-dimensional objects (Burke et al., 2012c; Deshmukh et al., 2012), and the PRC shares direct connections with both the LEC and distal CA1 and is known to be affected by normal aging (Burke et al., 2010, 2011b, 2012a,b; Ryan et al., 2012). All of these factors are consistent with the observation in the present report of less differentiated cell activity patterns along the proximodistal axis of CA1 in advanced age.

Finally, the current data reveal that there is a significant correlation between path overlap and similarity scores during the AA condition in proximal CA1 but not in distal CA1. This finding corroborates the idea that proximal CA1 pyramidal cells are more strongly spatially responsive than are those in distal CA1 (Henriksen et al., 2010, and supports the hypothesis that distal CA1 cells are more influenced by objects within the environment (Burke et al., 2011a). Moreover, it has been reported that neurons in distal CA1 have more dispersed firing fields and are more likely to have multiple place fields within an environment than do proximal CA1 neurons (Henriksen et al., 2010). Thus, a rat with a low path overlap between two exploratory epochs is more likely to traverse place fields from the same distal CA1 neuron population between epochs compared with the more spatially constrained proximal CA1 cells.

Along these lines, the significant relationship between behavioral trajectory overlap in the AA condition and the strength of the similarity score in proximal CA1 suggests that some of the variance in similarity scores is attributable to behavioral differences across identical sessions. Because the older rats showed reduced path overlap compared with adults in the AA condition $(\sim 0.9$ for adult, $\sim 0.8$ for old; Fig. 7), the observed age group difference in $A r c$ expression compartment patterns is most likely attributable to the difference in behavior between epochs (Fig. 10). In open arenas, rats do not exhibit stereotyped trajectories. Thus, it is possible to obtain data that suggest remapping when the change in place field expression between epoch 1 and 2 is actually attributable to different place fields crossed within the apparatus. The idea that the similarity score difference in proximal CA1 of old rats is attributable to differences in behavior rather than age is consistent with at least two earlier Arc catFISH investigations that observed map stability between epochs in old rats (Small et al., 2004; Penner et al., 2011). It is possible that, in those studies, adult and old rats showed more equivalent exploratory behaviors within the AA epochs. Although this provides an explanation of the difference between those results and the results reported here, path overlap was not measured in either of those experiments. It also is important to note that the stability of map retrieval in CA1 for old rats in these catFISH studies is not, on the surface, consistent with the electrophysiological observation of remapping in CA1 ensembles. At least two sources for these differences can be suggested: (1) the electrophysiological remapping was observed under different behavioral conditions, and (2) the extent of sampling was very different (Barnes et al., 1997). Because remapping is a stochastic event in any given animal, it is simply less probable to capture a remapping event with the catFISH method. The behavior in the electrophysiological studies involved traversal of a constrained linear track, with many repeated place field entries and over many days, making it more probable that such an event could be captured. In Arc catFISH experiments, only a single session on a single day is tested. Regardless of method used, it is clearly critical to control for differences in the exact path traversed within a single environment, because this variable can alter the active proximal CA1 neuron population.

The present study shows that there are differences between the responsiveness of distal versus proximal CA1 pyramidal cells to changes in spatial context in the presence of objects. Distal CA1 neurons do not remap when objects are stable, but the spatial context changes. Future models of CA1 contributions to the functional computations performed by different hippocampal subregions will need to consider this greater complexity along the CA1 proximodistal axis. Moreover, it is conceivable that distal and proximal CA1 are extensions of two distinct cortical networks that are engaged in different components of memory. Distal CA1 could participate in an anterior temporal system, which includes the PRC and LEC that relays representations of specific 
stimuli. In contrast, proximal CA1 is likely part of a posterior medial system involving the MEC, postrhinal cortex (parahippocampal in primates), and the retrosplenial cortex that are critical for processing information related to spatial context (Ranganath and Ritchey, 2012). The age-associated changes in activity patterns of neural ensembles across CA1 is consistent with alterations in both of these systems and may partly account for observed memory deficits in aging (for review, see Rosenzweig and Barnes, 2003; Wilson et al., 2006). Understanding these alterations is crucial for gaining insight into the human aging process and points to possible mechanisms underlying cognitive changes associated with normative aging.

\section{References}

Amaral D, Witter M (1995) Hippocampal formation. In: The rat nervous system, Ed 2 (Paxinos G, ed), pp 443-486. San Diego: Academic.

Barnes CA, Rao G, McNaughton BL (1996) Functional integrity of NMDAdependent LTP induction mechanisms across the lifespan of F-344 rats. Learn Mem 3:124-137. CrossRef Medline

Barnes CA, Suster MS, Shen J, McNaughton BL (1997) Multistability of cognitive maps in the hippocampus of old rats. Nature 388:272-275. CrossRef Medline

Burke SN, Wallace JL, Nematollahi S, Uprety AR, Barnes CA (2010) Pattern separation deficits may contribute to age-associated recognition impairments. Behav Neurosci 124:559-573. CrossRef Medline

Burke SN, Maurer AP, Nematollahi S, Uprety AR, Wallace JL, Barnes CA (2011a) The influence of objects on place field expression and size in distal hippocampal CA1. Hippocampus 21:783-801. CrossRef Medline

Burke SN, Wallace JL, Hartzell AL, Nematollahi S, Plange K, Barnes CA (2011b) Age-associated deficits in pattern separation functions of the perirhinal cortex: a cross-species consensus. Behav Neurosci 125:836-847. CrossRef Medline

Burke SN, Ryan L, Barnes CA (2012a) Characterizing cognitive aging of recognition memory and related processes in animal models and in humans. Front Aging Neurosci 4:15. CrossRef Medline

Burke SN, Hartzell AL, Lister JP, Hoang LT, Barnes CA (2012b) Layer V perirhinal cortical ensemble activity during object exploration: a comparison between young and aged rats. Hippocampus 22:2080-2093. CrossRef Medline

Burke SN, Maurer AP, Hartzell AL, Nematollahi S, Uprety A, Wallace JL, Barnes CA (2012c) Representation of 3-dimensional objects by the rat perirhinal cortex. Hippocampus 22:2032-2044. CrossRef Medline

Deshmukh SS, Knierim JJ (2011) Representation of non-spatial and spatial information in the lateral entorhinal cortex. Front Behav Neurosci 5:69. CrossRef Medline

Deshmukh SS, Johnson JL, Knierim JJ (2012) Perirhinal cortex represents nonspatial, but not spatial, information in rats foraging in the presence of objects: comparison with lateral entorhinal cortex. Hippocampus 22: 2045-2058. CrossRef Medline

Ennaceur A, Delacour J (1988) A new one-trial test for neurobiological studies of memory in rats. 1: Behavioral data. Behav Brain Res 31:47-59. CrossRef Medline

Fyhn M, Molden S, Witter MP, Moser EI, Moser MB (2004) Spatial representation in the entorhinal cortex. Science 305:1258-1264. CrossRef Medline

Gallagher M, Burwell R, Burchinal M (1993) Severity of spatial learning impairment in aging: development of a learning index for performance in the Morris water maze. Behav Neurosci 107:618-626. CrossRef Medline

Guzowski JF, McNaughton BL, Barnes CA, Worley PF (1999) Environmentspecific expression of the immediate-early gene Arc in hippocampal neuronal ensembles. Nat Neurosci 2:1120-1124. CrossRef Medline

Guzowski JF, McNaughton BL, Barnes CA, Worley PF (2001) Imaging neural activity with temporal and cellular resolution using FISH. Curr Opin Neurobiol 11:579-584. CrossRef Medline

Henriksen EJ, Colgin LL, Barnes CA, Witter MP, Moser MB, Moser EI
(2010) Spatial representation along the proximodistal axis of CA1. Neuron 68:127-137. CrossRef Medline

Komorowski RW, Manns JR, Eichenbaum H (2009) Robust conjunctive item-place coding by hippocampal neurons parallels learning what happens where. J Neurosci 29:9918-9929. CrossRef Medline

Lee I, Yoganarasimha D, Rao G, Knierim JJ (2004) Comparison of population coherence of place cells in hippocampal subfields CA1 and CA3. Nature 430:456-459. CrossRef Medline

Manns JR, Eichenbaum H (2009) A cognitive map for object memory in the hippocampus. Learn Mem 16:616-624. CrossRef Medline

Morris R (1984) Developments of a water-maze procedure for studying spatial learning in the rat. J Neurosci Methods 11:47-60. CrossRef Medline

Morris RG, Garrud P, Rawlins JN, O'Keefe J (1982) Place navigation impaired in rats with hippocampal lesions. Nature 297:681-683. CrossRef Medline

O'Keefe J, Conway DH (1978) Hippocampal place units in the freely moving rat: why they fire where they fire. Exp Brain Res 31:573-590. Medline

O’Keefe J, Dostrovsky J (1971) The hippocampus as a spatial map. Preliminary evidence from unit activity in the freely-moving rat. Brain Res 34: 171-175. CrossRef Medline

O'Keefe J, Nadel L (1978) The hippocampus as a cognitive map. Oxford: Claredon.

Penner MR, Roth TL, Chawla MK, Hoang LT, Roth ED, Lubin FD, Sweatt JD, Worley PF, Barnes CA (2011) Age-related changes in Arc transcription and DNA methylation within the hippocampus. Neurobiol Aging 32: 2198-2210. CrossRef Medline

Ranganath C, Ritchey M (2012) Two cortical systems for memory-guided behaviour. Nat Rev Neurosci 13:713-726. CrossRef Medline

Rapp PR, Gallagher M (1996) Preserved neuron number in the hippocampus of aged rats with spatial learning deficits. Proc Natl Acad Sci U S A 93:9926-9930. CrossRef Medline

Rosenzweig ES, Barnes CA (2003) Impact of aging on hippocampal function: plasticity, network dynamics, and cognition. Prog Neurobiol 69: 143-179. CrossRef Medline

Ryan L, Cardoza JA, Barense MD, Kawa KH, Wallentin-Flores J, Arnold WT, Alexander GE (2012) Age-related impairment in a complex object discrimination task that engages perirhinal cortex. Hippocampus 22: 1978-1989. CrossRef Medline

Sava S, Markus EJ (2008) Activation of the medial septum reverses agerelated hippocampal encoding deficits: a place field analysis. J Neurosci 28:1841-1853. CrossRef Medline

Schmidt B, Satvat E, Argraves M, Markus EJ, Marrone DF (2012) Cognitive demands induce selective hippocampal reorganization: $\operatorname{Arc}$ expression in a place and response task. Hippocampus 22:2114-2126. CrossRef Medline

Small SA, Chawla MK, Buonocore M, Rapp PR, Barnes CA (2004) Imaging correlates of brain function in monkeys and rats isolates a hippocampal subregion differentially vulnerable to aging. Proc Natl Acad Sci U S A 101:7181-7186. CrossRef Medline

Thompson LT, Best PJ (1990) Long-term stability of the place-field activity of single units recorded from the dorsal hippocampus of freely behaving rats. Brain Res 509:299-308. CrossRef Medline

Vazdarjanova A, Guzowski JF (2004) Differences in hippocampal neuronal population responses to modifications of an environmental context: evidence for distinct, yet complementary, functions of CA3 and CA1 ensembles. J Neurosci 24:6489-6496. CrossRef Medline

Vazdarjanova A, Ramirez-Amaya V, Insel N, Plummer TK, Rosi S, Chowdhury S, Mikhael D, Worley PF, Guzowski JF, Barnes CA (2006) Spatial exploration induces ARC, a plasticity-related immediate-early gene, only in calcium/calmodulin-dependent protein kinase II-positive principal excitatory and inhibitory neurons of the rat forebrain. J Comp Neurol 498: 317-329. CrossRef Medline

Wilson IA, Gallagher M, Eichenbaum H, Tanila H (2006) Neurocognitive aging: prior memories hinder new hippocampal encoding. Trends Neurosci 29:662-670. CrossRef Medline

Zhao M, Choi YS, Obrietan K, Dudek SM (2007) Synaptic plasticity (and the lack thereof) in hippocampal CA2 neurons. J Neurosci 27:1202512032. CrossRef Medline 